\title{
Las alianzas interempresariales en el estado de Mérida, Venezuela ${ }^{1}$
}

Marlene Peñaloza

Facultad de Ciencias Económicas y Sociales, Universidad de Los Andes, Venezuela pemarle@hotmail.com

\section{Resumen}

Frente a los retos de los mercados mundializados reaparecen las alianzas o acuerdos interempresariales, que son frecuentemente utilizados por empresas de gran tamaño aun cuando no excluye a las de pequeñas dimensiones. Esta investigación se desarrolló con el propósito de caracterizar las alianzas existentes entre las empresas del estado de Mérida, Venezuela. Para tal efecto, se aplicó un cuestionario a 228 propietarios/gerentes mediante entrevista personal y se contrastó una hipótesis de trabajo. El análisis de los resultados indica baja propensión a establecer alianzas, predominio de las alianzas de naturaleza vertical y en el área de marketing. Prevalecen las none equity alliances y las coaliciones como formas de cooperación. Las formas legales más utilizadas son el outsourcing y la franquicia. Entre los motivos para no aliarse resaltan la falta de confianza y el temor a perder independencia. Finalmente, se encontró una relación moderada entre tamaño relativo de la empresa y la conformación de alianzas.

Palabras clave: alianzas interempresariales, acuerdos, cooperación, empresas, competencia. 


\title{
Interenterprise alliances in Mérida State, Venezuela
}

\begin{abstract}
Faced with the challenges of globalized markets alliances or interenterprise agreements reappear, frequently used by large size companies even though small size ones are not excluded. This investigation was developed in order to characterize the existing alliances between the companies of Mérida state (Venezuela). To this end, a questionnaire was applied to 228 proprietors/managers by means of a personal interview and a work hypothesis was contrasted. Analysis of the results indicates a low propensity to establish alliances, predominance of the alliances of a vertical nature and in the marketing area. None equity alliances and cooperation coalitions prevail. Most used legal forms are outsourcing and franchising. Among the reasons not to ally lack of confidence and fear of losing independence stand out. Finally, a moderate relation between relative size of the company and the conformation of alliances was found.
\end{abstract}

Keywords: interenterprise alliances, agreements, cooperation, companies, competition.

\section{Introducción}

Las nuevas tendencias mundiales apuntan hacia una dinámica competitiva que requiere de nuevos comportamientos y en la cual las alianzas entre empresas tienen un papel principal. Tales acuerdos suelen ser considerados una vía rápida y menos costosa para el desarrollo de la capacidad innovadora y tecnológica, mejorar el acceso a los mercados y la competitividad, compartir riesgos y reducir costos, entre otros aspectos (Casani, 1995; Urra, 1998). En consecuencia, la estrategia de la colaboración empresarial pareciera adecuada tanto para las empresas de mayor tamaño relativo como para las medianas y pequeñas (Porter y Fuller, 1988; Rosales, 1997).

En Venezuela, el tema ha sido poco transitado con excepción de la industria petrolera. En cuanto al estado de Mérida, cuyo parque empresarial está conformado básicamente por micro y pequeñas empresas dedicadas al comercio y a los servicios, pareciera estar un poco lejos de esta tendencia.

${ }^{1}$ Proyecto financiado Por el CDCHTA-ULA 
Dada la importancia del tema, la gran diversidad de tipos de alianzas empresariales, pero al mismo tiempo la heterogeneidad en la terminología empleada y los diferentes enfoques que tratan de explicar por qué las empresas cooperan entre sí, en esta investigación se revisa grosso modo los fundamentos teóricos que sustentan los acuerdos entre empresas, su taxonomía y tipología, para luego abordar el estudio de los objetivos y las distintas formas asociativas seguidas por las empresas del estado de Mérida. A tal efecto se llevó a cabo un estudio de campo mediante la técnica de entrevistas a los gerentes y/o propietarios de 228 empresas, planteándose además una hipótesis de investigación que fue contrastada mediante la prueba Chi-cuadrado. Las variables en estudio se analizan mediante gráficas y/o cuadros de frecuencia.

\section{Consideraciones generales}

Ante la intensa competencia, consumidores mejor informados, más selectivos y menos leales, niveles de innovación creciente y alta competitividad, las firmas están en la búsqueda de nuevas estructuras y estrategias que les permitan responder con rapidez a las demandas del entorno.

Entre otras formas de organización y de gestión empresarial han reaparecido en los últimos años los acuerdos o alianzas. Dichos acuerdos como estrategia empresarial no son nuevos pues han sido utilizados en otros tiempos por la gran empresa ${ }^{2}$ (Valdaliso y López, 2003). Sin embargo, investigaciones empíricas en el nivel mundial señalan que las nuevas tendencias apuntan hacia una dinámica competitiva que requiere de nuevos comportamientos dentro de las cuales la cooperación tiene un papel principal (Hamel, Doz y Prahalad, 1989; Jorde y Teece, 1989; Porter y Fuller, 1988).

Igualmente, los ejemplos de países del sudeste asiático con sus redes y conglomerados, así como las experiencias en Italia (Emilia Romagna), en Estados Unidos (Silicon Valley) y en Alemania (Baden-Wuttember) han llamado la atención mundial. También en América Latina, cuyo tejido empresarial está conformado básicamente por Pyme (FUNDES, 2002), es reconocida las experiencias de articulación productiva en países como Chile y Brasil (Piñones, 2004).

\footnotetext{
${ }^{2}$ En el período de auge de las empresas multinacionales, la cooperación se consideró como una opción válida cuando no le era fácil entrar a mercados foráneos.
} 
En cuanto a su fundamentación teórica, diferentes estudios han intentado explicar las razones por las cuales las empresas buscan la colaboración de otras; esta diversidad de estudios se caracteriza por su dispersión y parcialidad, pues han sido realizados desde ángulos diferentes y por diversas disciplinas. No obstante, los distintos enfoques pueden ser agrupados bajo tres perspectivas teóricas: económica, estratégica y organizativa (Casani, 1995; Menguzzato, 1995), las cuales se resumen en el cuadro 1.

\section{Cuadro 1}

Aspectos básicos en los enfoques sobre los acuerdos interempresariales

\begin{tabular}{|c|c|c|}
\hline Enfoque económico & Enfoque estratégico & Enfoque organizativo \\
\hline $\begin{array}{l}\text { - } \begin{array}{l}\text { Modelo estructura- } \\
\text { comportamiento- } \\
\text { resultados. }\end{array} \\
\text { - Límites de la } \\
\text { organización a partir de } \\
\text { la función de producción. } \\
\text { - Actividad económica } \\
\text { regida por el mercado. } \\
\text { - Actividad económica } \\
\text { basada en la autoridad y } \\
\text { la jerarquía. } \\
\text { - Reducir costes de } \\
\text { transacción. } \\
\text { - Formas híbridas de } \\
\text { organización. } \\
\text { (Coase, 1937; Eggertsson, } \\
\text { 1995; Mason, 1957) }\end{array}$ & $\begin{array}{l}\text { - Teoría de la } \\
\text { internacionalización de } \\
\text { la producción. } \\
\text { - Mecanismo de estrategia } \\
\text { empresarial. } \\
\text { - Asociación mediante } \\
\text { fusiones, adquisiciones o } \\
\text { co-inversiones } \\
\text { - Fluidez y flexibilidad. } \\
\text { - Acuerdos de } \\
\text { cooperación: } \\
\text { Joint ventures (de } \\
\text { producción, de I\&D), } \\
\text { outsourcing, licensing, } \\
\text { redes de empresas, } \\
\text { cluster industriales. } \\
\text { (Hamel Doz y Prahalad, } \\
\text { 1989; Porter y Fuller, 1988) }\end{array}$ & $\begin{array}{l}\text { - Teoría de los recursos y } \\
\text { capacidades. } \\
\text { - Activos basados en el } \\
\text { conocimiento. } \\
\text { - Conocimiento tácito. } \\
\text { - Aprendizaje cotidiano. } \\
\text { - Efecto experiencia. } \\
\text { - La cooperación como medio de } \\
\text { aprendizaje organizacional. }\end{array}$ \\
\hline
\end{tabular}

Con respecto a su terminología, es igualmente profuso lo que dificulta arribar a consensos, pues inclusive los términos se traslapan. Así, encontramos diferentes denominaciones como coaliciones (Porter y Fuller, 1988), asociatividad (Rosales, 1997), cooperación (Federación Andaluza de Municipios y Provincias, 2004) acuerdos de cooperación (Mariti y Smiley, 1983), alianzas estratégicas (Jorde y Teece, 1989) redes (Kochaztky, 2002). 
En cuanto a su conceptualización, tampoco existe consenso, pues las acepciones del término varían en función del enfoque teórico desde el cual se visualiza el acuerdo y de las diferentes maneras de formalizarlo. A continuación se enuncian algunas de sus acepciones (cuadro 2).

\section{Cuadro 2 \\ Definiciones}

\begin{tabular}{l|l}
\hline \multicolumn{1}{c|}{ Autor } & \multicolumn{1}{c}{ Definiciones } \\
\hline $\begin{array}{l}\text { T. Jorde y D. Teece } \\
\text { (1989) }\end{array}$ & $\begin{array}{l}\text { Una alianza estratégica puede ser definida como una relación bilateral } \\
\text { caracterizada por un compromiso entre dos o más empresas para alcanzar } \\
\text { una meta común. }\end{array}$ \\
$\begin{array}{l}\text { S. Martínez Fierro } \\
\text { (2001) }\end{array}$ & $\begin{array}{l}\text { Las alianzas estratégicas o los acuerdos de cooperación son relaciones } \\
\text { explícitas y deliberadas entre dos o más organizaciones independientes } \\
\text { que les permite compartir, durante un periodo de tiempo determinado y } \\
\text { de forma equitativa, recursos, capacidades y/o competencias, actuando en } \\
\text { común y coordinando las acciones objeto del acuerdo en cualquier ámbito } \\
\text { de su actuación, sin que exista entre ellas una relación de subordinación } \\
\text { para perseguir unos objetivos que individualmente no hubiesen logrado. }\end{array}$ \\
$\begin{array}{l}\text { Ma. Costa Campi } \\
\text { (1989) }\end{array}$ & $\begin{array}{l}\text { Los acuerdos implican, por un lado, el mantenimiento de la personalidad } \\
\text { jurídica independiente de las empresas, quedando por tanto excluidas } \\
\text { las fusiones, y por otro, el establecimiento de fórmulas de colaboración } \\
\text { en proyectos nacionales o trasnacionales de investigación, producción y } \\
\text { comercialización. }\end{array}$ \\
\hline
\end{tabular}

Con relación a su tipología, existen diferentes clasificaciones las que pueden adquirir múltiples formas en correspondencia con los criterios subyacentes y los objetivos que se persigan (cuadro 3 ).

Ante la problemática de su terminología, conceptualización y tipología, resulta pertinente hacer las siguientes precisiones:

- En esta investigación se utilizan como sinónimos los términos acuerdos, alianzas o cooperación aun cuando se reconoce que no siempre su alcance es el mismo.

- Los criterios para clasificar los acuerdos se han seleccionado considerando los de mayor uso en la literatura.

- Frente a la diversidad y el amplio espectro de relaciones que se recoge bajo el término, en este trabajo se entiende por acuerdos de cooperación toda 
alianza o asociación temporal o permanente que, de manera voluntaria y concertada, se establece entre dos o más empresas en alguna o varias áreas de actividad, generalmente formalizada y respaldada contractualmente, cuya búsqueda de complementariedad y metas comunes persigue como fin último la obtención de ventajas competitivas.

\section{Cuadro 3}

\section{Principales criterios para clasificar los acuerdos}

\begin{tabular}{l|l}
\hline 1. Naturaleza de los acuerdos & $\begin{array}{l}\text { Vertical } \\
\text { Horizontal }\end{array}$ \\
\hline 2. Campo de aplicación & $\begin{array}{l}\text { Cadena de valor } \\
\text { Áreas funcionales }\end{array}$ \\
\hline 3. Formas de los acuerdos & $\begin{array}{l}\text { Referente a la propiedad } \\
\text { Estructura contractual } \\
\text { Número de vínculos }\end{array}$ \\
\hline 4. Estructura legal de los & $\begin{array}{l}\text { Joint venture } \\
\text { Franquicia } \\
\text { Licencia } \\
\text { Outsourcing }\end{array}$ \\
& $\begin{array}{l}\text { Spin-off } \\
\text { Capital de riesgo }\end{array}$ \\
\hline
\end{tabular}

A partir de estas interrogantes se planteó una hipótesis de investigación la cual fue contrastada mediante la prueba Chi-cuadrado y el test de independencia:

$\mathrm{H}_{1}$ El tamaño de la empresa, medido en términos del número de trabajadores, condiciona las posibilidades de establecer alianzas: cuanto más grande es el tamaño, aumentan las posibilidades de establecer acuerdos de cooperación.

\section{Los acuerdos entre Pyme}

La evidencia empírica muestra que las empresas que participan en acuerdos asociativos disponen de una ventaja competitiva respecto a las que actúan en forma

\footnotetext{
${ }^{3}$ Joint-venture: es una nueva entidad surgida de un acuerdo entre dos firmas independientes, con personalidad jurídica independiente para la realización de las actividades objeto del acuerdo, aunque con intereses supeditados a las estrategias competitivas de los socios que la crearon. Ver a Federación Andaluza de Municipios y Provincias (2004).

${ }^{4}$ Outsourcing: acuerdo que consiste en la subcontratación de alguna actividad necesaria en el proceso de producir y comercializar un bien, pero que por distintas razones a la empresa le conviene subcontratar. Puede también decidirse subcontratar cuando se requiere incorporar nuevas tecnologías, e inclusive en la gestión de calidad (idem).

${ }^{5}$ Spin-off: son empresas que surgen del esfuerzo realizado por un grupo de trabajadores cualificados de otra empresa por lo general de gran tamaño, la cual apoya a la nueva empresa desde un principio y con la que posteriormente mantendrá la cooperación en el área de producción (ibídem).
} 
aislada por la eficiencia colectiva a las que son expuestas (Pietrobelli y Rabellotti, 2005). Ello induciría a pensar una alta propensión por parte de las empresas a celebrar acuerdos; no obstante, la literatura reseña con bastante frecuencia que las estrategias cooperativas son usualmente lejanas a las posibilidades e intereses de las empresas especialmente dentro de la categoría de Pyme, cuya tendencia es más bien a bregar solas, sobre todo en los países en desarrollo (CEPAL, 2007; Rosales, 1997).

Entre las razones que explicarían su escasa propensión a cooperar se mencionan, por lo general, el hecho de ser gestionadas bajo una cultura personalista, sin delegar funciones, con escasa planificación a largo plazo, el carácter personal o familiar, recelos para compartir conocimientos, formas de gestión, baja tecnología; falta de atractivo por su baja productividad (debido a su tamaño relativo), la poca calidad de sus productos, escaso capital, su ámbito local y regional, la desconfianza hacia otros empresarios, el miedo a perder la independencia y la poca cultura hacia la cooperación (CEPAL, 2007; Romero, 2004; OIT, 2004-2005;). Algunos de estos obstáculos pueden ser más aparentes que reales si son adecuadamente administrados.

Sin embargo, entre éstos existe un aspecto que pareciera ser clave para la conformación de alianzas y en torno a los cuales gira una interesante discusión ${ }^{6}$ que por razones de espacio no es posible abordarlos en un artículo de esta naturaleza:

La dimensión de la empresa, por la controvertida relación entre tamaño de la firma-productividad-ventajas competitivas-capacidad de innovación (Camisón, 2000; OIT, 2004-2005).

\section{La empresa emeritense ${ }^{7}$}

El parque empresarial del estado de Mérida ${ }^{8}$ está conformado básicamente por micro, pequeñas y medianas empresas en gran medida dedicadas al comercio y a los

\footnotetext{
${ }^{6}$ También menciona la falta de confianza en las alianzas (Berry, 1997; Vázquez, 2005), sobre todo en América Latina donde a decir de Berry (1997) el hombre de negocios es altamente individualista y poco propenso a confiar y a cooperar con sus pares.

${ }^{7}$ Emeritense es el gentilicio del estado de Mérida, Venezuela.

${ }^{8}$ El estado de Mérida está ubicado en el occidente del país, aproximadamente a $800 \mathrm{~km}$ de la capital e integrado por 23 municipios. El Instituto Nacional de Estadística (INE) ha situado la población de la entidad para 2007 en 846830 personas. El estado ocupa el décimo lugar en tamaño en relación con el resto de entidades federales del país.
} 
servicios, surgidas fundamentalmente para dar respuesta a la creciente demanda por parte de la población, con escaso capital, casi o ninguna especialización y bajo nivel organizativo, tecnológico y gerencial (CORPOANDES, 1990). Además, están geográficamente aisladas de los principales centros de desarrollo del país, en una topografía accidentada que le dificulta la provisión de insumos y la colocación de sus productos fuera de su ámbito natural, con una demanda local pequeña y unas características propias de la idiosincrasia del merideño que marca su desempeño empresarial (Ortiz, 2000).

Ante un parque empresarial conformado básicamente por negocios de pequeñas dimensiones es factible preguntarse ¿Cuál es el su comportamiento frente a la cooperación empresarial? ¿Cuál es la incidencia del tamaño relativo de la empresa en la conformación de acuerdos?

\section{Objetivo de la investigación}

Caracterizar las relaciones existentes entre las empresas localizadas en el ámbito del estado de Mérida desde la perspectiva de la conformación de acuerdos de alianzas interempresariales.

\section{Metodología para el análisis del sector}

Para identificar los mecanismos de cooperación existente entre las empresas del estado de Mérida, en una primera fase se revisó literatura sobre el tema. La segunda fase consistió en un estudio de campo mediante la técnica de entrevista personal realizada a propietarios o en su defecto a gerentes de empresas legalmente registradas en las alcaldías de los municipios que integran a Mérida. ${ }^{9}$ Para tal efecto, se aplicó un cuestionario de 32 preguntas estructuradas en dos secciones referidas a la identificación de la empresa y a la indagación del tema objeto de estudio.

El primer bloque de preguntas estuvo dirigido a la caracterización de las empresas en cuanto a actividad económica, tiempo de funcionamiento y número de trabajadores; esto último permitió categorizar a las empresas por estratos de ocupación. Un segundo bloque de reactivos se orientó al conocimiento de las particularidades

\footnotetext{
${ }^{9}$ Se seleccionaron 18 de los 23 municipios que conforman el estado Mérida, cinco de éstos son netamente rurales y ninguno de sus pequeños centros poblados alcanza la categoría de urbanos (Méndez, 2003). Asimismo, fue necesario depurar los listados por cuanto hubo que excluir parte de las microempresas como bodegas y kioscos, venta de víveres, buhonería y agencias de loterías, registros no válidos o repetidos.
} 
de los acuerdos realizados entre las empresas merideñas. Finalmente, al término del cuestionario se preguntó sobre las motivaciones para no asociarse.

La muestra fue de 18278 empresas; el tipo de muestreo, aleatorio simple con un tamaño de la muestra para estimar la Proporción P (Scheaffer, Mendenhall y Ott, 1991) de empresas con alianzas utilizando el criterio de varianza máxima $\mathrm{P}=0.5$ para un error de estimación de B igual a $\pm 5.3 \%$ y un nivel de confianza de $95 \%$, en la que resultó $n=228$. La estimación del tamaño de la muestra se hizo bajo la restricción de costo fijo por contar con un presupuesto predeterminado.

Las empresas se clasificaron en atención con lo dispuesto en la Ley de Promoción a la Pequeña y Mediana Industria y Unidades de Promoción Social: micro: 1-10 empleados; pequeña: 11-50; mediana: 51-100; grande: 101 en adelante. La muestra resultó estar conformada mayoritariamente por empresas en la categoría de Micro; en menor proporción resultaron las pequeñas y medianas; en la categoría de grandes en la muestra sólo se encontraron dos empresas.

\section{Análisis de resultados}

Para el análisis se incluyen los bloques que contienen las preguntas a partir de las cuales se contrastaron las hipótesis, mostrándose los análisis mediante gráficas, cuadros de frecuencias y/o cuadros ${ }^{10}$, los cuales han sido elaborados con el propósito de ilustrar los resultados obtenidos de la prueba empírica aplicada a 228 propietarios o gerentes.

La pregunta: ¿Ha realizado algún tipo de alianza? (cuadro 4) se aplicó a todos los sujetos de estudio, lo cual permitió agrupar las respuestas en dos categorías: Con alianza y Sin alianza.

\section{Cuadro 4}

¿Ha realizado algún tipo de alianza?

\begin{tabular}{l|c|c|c|c}
\hline $\begin{array}{c}\text { ¿Tiene } \\
\text { alianza? }\end{array}$ & Frecuencia & Porcentaje & $\begin{array}{c}\text { Porcentaje } \\
\text { válido }\end{array}$ & $\begin{array}{c}\text { Porcentaje } \\
\text { acumulado }\end{array}$ \\
\hline Sí & 57 & 25.0 & 25.0 & 25.0 \\
\hline No & 171 & 75.0 & 75.0 & 100.0 \\
\hline Total & 228 & 100.0 & 100.0 & \\
\hline
\end{tabular}

\footnotetext{
${ }^{10}$ Los cuadros estadísticos se construyeron tomando las frecuencias y los porcentajes de las cuadros estadísticos correspondientes a cada uno de los ítems de las preguntas consideradas en el cuestionario aplicado.
} 
Como puede verse en el cuadro 4, de 228 empresas consultadas sólo 57 empresas (25\%) han realizado algún tipo de alianza, lo que indica baja disposición de las empresas locales para cooperar con sus pares, y viene a reforzar lo señalado en la literatura como es la tendencia de las Pyme a actuar aisladamente (CEPAL, 2007; Rosales, 1997).

Las siguientes preguntas se aplicaron a todos los encuestados: Sector económico al que pertenece la empresa (cuadro 5), Antigüedad de la empresa (cuadro 6), Número de trabajadores (gráfica 1).

En cuanto al sector económico (cuadro 2) predomina el comercio (42.9\%), seguido por servicios (38.9\%), industria (15.9\%) y construcción $(2.2 \%)$.

\section{Cuadro 5}

Sector económico

\begin{tabular}{|c|c|c|c|c|}
\hline Sector económico & Frecuencia & Porcentaje & $\begin{array}{c}\text { Porcentaje } \\
\text { válido }\end{array}$ & $\begin{array}{l}\text { Porcentaje } \\
\text { acumulado }\end{array}$ \\
\hline Pesca & 1 & .4 & .4 & .4 \\
\hline Industrias manufactureras & 36 & 15.8 & 15.8 & 16.2 \\
\hline Suministro de gas & 1 & .4 & .4 & 16.7 \\
\hline Construcción & 5 & 2.2 & 2.2 & 18.9 \\
\hline $\begin{array}{l}\text { Comercio al por mayor y al por } \\
\text { menor }\end{array}$ & 97 & 42.5 & 42.5 & 61.4 \\
\hline Servicio de hoteles y restaurantes & 54 & 23.7 & 23.7 & 85.1 \\
\hline $\begin{array}{l}\text { Servicios de transporte, } \\
\text { almacenamiento y comunicaciones }\end{array}$ & 3 & 1.3 & 1.3 & 86.4 \\
\hline $\begin{array}{l}\text { Servicios de intermediación } \\
\text { financieros }\end{array}$ & 3 & 1.3 & 1.3 & 87.7 \\
\hline $\begin{array}{l}\text { Servicios inmobiliarios, } \\
\text { empresariales y de alquiler }\end{array}$ & 8 & 3.5 & 3.5 & 91.2 \\
\hline Servicios sociales y de salud & 2 & .9 & .9 & 92.1 \\
\hline $\begin{array}{l}\text { Servicios comunitarios, sociales y } \\
\text { personales }\end{array}$ & 18 & 7.9 & 7.9 & 100.0 \\
\hline Total & 228 & 100.0 & 100.0 & \\
\hline
\end{tabular}

Por lo que respecta a los años que lleva funcionando la empresa (cuadro 6), casi el 60\% de las empresas merideñas tienen 10 años o más de funcionamiento, o sea, que su parque empresarial en general ha logrado mantenerse en el tiempo. 


\section{Cuadro 6}

\section{Antigüedad de la empresa}

\begin{tabular}{l|c|c|c|c}
\hline $\begin{array}{c}\text { Antigüedad } \\
\text { de la empresa }\end{array}$ & Frecuencia & Porcentaje & $\begin{array}{c}\text { Porcentaje } \\
\text { válido }\end{array}$ & $\begin{array}{c}\text { Porcentaje } \\
\text { acumulado }\end{array}$ \\
\hline De 0 a 4 años & 35 & 15.4 & 15.4 & 15.4 \\
\hline De 5 a 9 años & 59 & 25.9 & 25.9 & 41.3 \\
\hline $\begin{array}{l}\text { De 10 años } \\
\text { y más }\end{array}$ & 134 & 58.7 & 58.7 & 100.0 \\
\hline Total & 228 & 100.0 & 100.0 & \\
\hline
\end{tabular}

Igual tratamiento se dio a la pregunta sobre el número de empleados, es decir, se aplicó a todas las empresas encuestadas, lo que permitió su clasificación por estratos de ocupación (gráfica 1).

\section{Gráfica 1}

\section{Distribución de las empresas por estratos de ocupación}

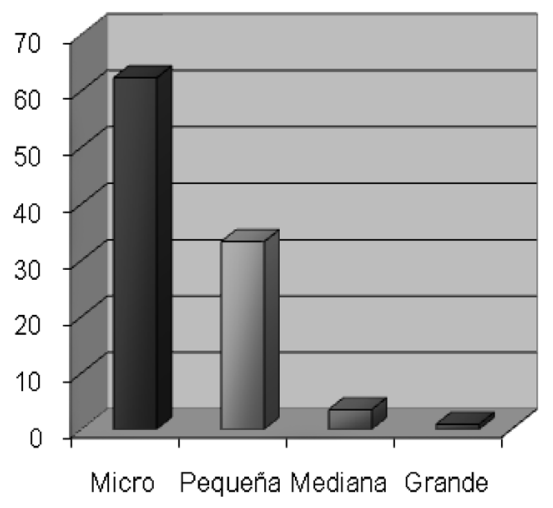

$$
\begin{aligned}
& \text { 口Micro } \\
& \text { 口Pequeña } \\
& \square \text { Mediana } \\
& \square G \text { rande }
\end{aligned}
$$

\section{Estratos de ocupación}

El siguiente bloque de preguntas está referido a la caracterización de las alianzas celebradas entre las empresas merideñas, por lo que sólo fueron consultados los que han realizado alianzas.

Al indagar sobre la Naturaleza de los acuerdos, el $78.9 \%$ de las alianzas son de tipo vertical y $19.3 \%$ de carácter horizontal (gráfica 2). Estos acuerdos entre empresas ubicadas en diferentes sectores bien "aguas arriba" o "aguas abajo" son comunes entre Pyme en la búsqueda de complementariedades. Por tanto, su predominio entre en el ámbito local es comprensible ante las limitaciones y carencias 
de las empresas emeritenses para proveerse de insumos de calidad o contar con canales de distribución eficientes.

\section{Gráfica 2}

Naturaleza de los acuerdos

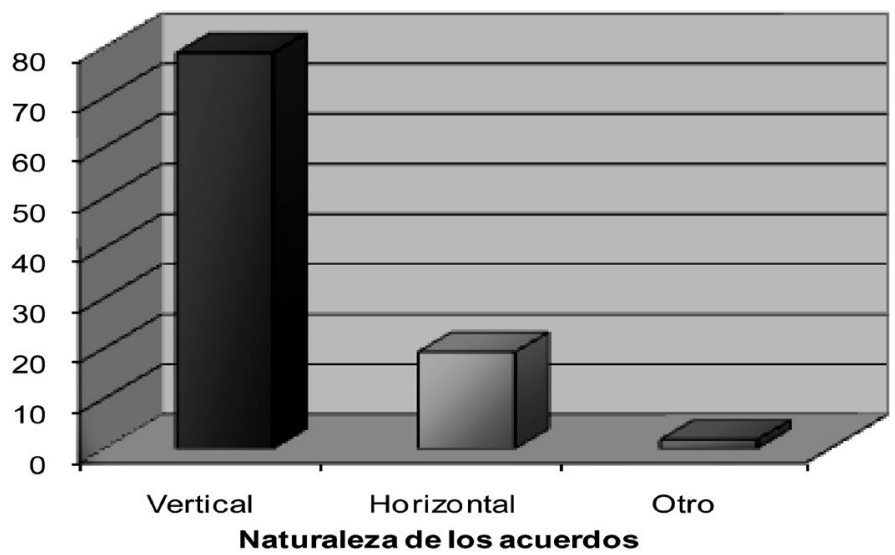

A la pregunta Campo de aplicación de los acuerdos (cuadro 7), predomina la cooperación comercial (89.5\%) también llamada de marketing, ventas y servicios, confirmándose lo apuntado por Porter y Fuller (1988) de ser una de las áreas preferidas por las Pyme para celebrar acuerdos. Le sigue de lejos operación y logística (24.6\%), actividad múltiple - incluye acuerdos en diversas áreas - (17.5\%), las de desarrollo tecnológico y cooperación financiera son las menos sujetas a acuerdos (12.3\%).

\section{Cuadro 7}

\section{Campo de aplicación de los acuerdos}

\begin{tabular}{l|l|l|l|l|l|l}
\hline Campo de aplicación de los acuerdos & \multicolumn{2}{|c|}{ Sí } & \multicolumn{2}{c|}{ No } & \multicolumn{2}{c}{ Total } \\
\hline & f* & \% & F & \% & f & \% \\
\hline Desarrollo tecnológico & 7 & 12.3 & 50 & 87.7 & 57 & 100 \\
\hline Operaciones y logística & 14 & 24.6 & 43 & 75.4 & 57 & 100 \\
\hline Marketing, ventas y servicio & 54 & 94.7 & 3 & 5.3 & 57 & 100 \\
\hline Actividad múltiple & 10 & 17.5 & 47 & 82.5 & 57 & 100 \\
\hline Cooperación comercial & 54 & 94.7 & 3 & 5.3 & 57 & 100 \\
\hline Cooperación financiera & 7 & 12.3 & 50 & 87.7 & 57 & 100 \\
\hline Cooperación tecnológica & 7 & 12.3 & 50 & 87.7 & 57 & 100 \\
\hline
\end{tabular}

Nota: Este cuadro se construyó tomando las frecuencias y los porcentajes de los cuadros estadísticos correspondientes a cada uno de los ítems de la pregunta $\mathrm{N}^{\circ} 18$ (de respuesta múltiple) del cuestionario aplicado.

* la f (s) indican la frecuencia con que se repite el fenómeno. 
Al preguntar sobre las Formas de los acuerdos ${ }^{11}$ se determinó que se conforman atendiendo a las formas de propiedad, a la estructura contractual y al número de vínculos. A su vez cada una de éstas se refleja en una diversidad de figuras, como puede denotarse a continuación:

La propiedad: No es usual para los empresarios merideños hacer alianzas que den origen a un joint venture (otra empresa) pues sólo el 10.5\% contestó afirmativamente. Lo mismo se observa para las equity strategic alliances, que pueden tomar muchas formas como holdings, consorcios y los propios joint ventures y que solamente se dan en $8.8 \%$ de los casos. Este comportamiento tiende a cambiar cuando se trata de acuerdos entre firmas socias no equitativas (none equity strategic alliances) que se presentan en el 29.8\% de los casos (García 1993; Koschatzky, 2002; Mariti y Smiley, 1983).

La estructura contractual: La figura predominante son los acuerdos de intercambio $(86.0 \%)$, seguida de las coaliciones $(70.2 \%)$ y, de lejos, por los joint ventures $(10.5 \%)$, los consorcios (10.5\%) y las participaciones accionarias minoritarias $(8.8 \%)$.

El número de vínculos: Las más frecuentes son las redes verticales (78.9\%), los que se refieren a sin alta concentración de capital (94.7\%) y las redes sencillas $(52.6 \%)$.

\footnotetext{
${ }^{11}$ Para una mejor comprensión Joint Ventures (definida anteriormente). Equity strategic alliances: acuerdos entre empresas comunes (Marity y Smiley, 1983). None equity strategic alliances: consisten en acuerdos entre firmas socias sin afiliación (Marity y Smiley, 1983). Cluster: agrupaciones de empresas (principalmente medianas y pequeñas) e instituciones interconectadas en un campo particular próximo, geográficamente unidas por prácticas comunes y complementarias, que se interrelacionan para el intercambio de bienes, información, recursos humanos, comercialización y otras funciones empresariales. Los keiretsus representan un conglomerado de empresas pertenecientes a distintos sectores económicos (banca, industria, servicios) y en una variedad de mercados (capital, artículos básicos, componentes) coordinados entre sí a través de vínculos patrimoniales comunes y de grupos de trabajos gerenciales (Freeman, 1993; Keegan y Green, 1998).
} 


\section{Cuadro 8}

\section{Formas de los acuerdos}

\begin{tabular}{|c|c|c|c|c|c|c|c|c|}
\hline \multirow[t]{2}{*}{ Formas de cooperación } & \multicolumn{2}{|c|}{ Sí } & \multicolumn{2}{|c|}{ No } & \multicolumn{2}{|c|}{ Ns/nc } & \multicolumn{2}{|c|}{ Total } \\
\hline & $f *$ & $\%$ & f & $\%$ & $\mathbf{F}$ & $\%$ & f & $\%$ \\
\hline \multicolumn{9}{|l|}{ Referente a la propiedad } \\
\hline Joint ventures & 6 & 10.5 & 51 & 89.5 & - & - & 57 & 100 \\
\hline Equity strategic alliances & 5 & 8.8 & 45 & 78.9 & 7 & 12.3 & 57 & 100 \\
\hline None equity strategic alliances & 17 & 29.8 & 31 & 54.4 & 9 & 15.8 & 57 & 100 \\
\hline \multicolumn{9}{|l|}{ Estructura contractual } \\
\hline Consorcios & 6 & 10.5 & 49 & 86.0 & 2 & 3.5 & 57 & 100 \\
\hline Coaliciones & 40 & 70.2 & 14 & 24.6 & 3 & 5.3 & 57 & 100 \\
\hline Joint ventures & 6 & 10.5 & 49 & 86.0 & - & - & 57 & 100 \\
\hline Acuerdos de intercambio & 49 & 86.0 & 6 & 10.5 & 2 & 3.5 & 57 & 100 \\
\hline $\begin{array}{l}\text { Participaciones accionariales } \\
\text { minoritarias }\end{array}$ & 5 & 8.8 & 46 & 80.7 & 6 & 10.5 & 57 & 100 \\
\hline \multicolumn{9}{|l|}{ Número de vínculos } \\
\hline Redes verticales & 45 & 78.9 & 12 & 21.1 & - & - & 57 & 100 \\
\hline Redes horizontales* & 11 & 17.5 & 46 & 80.7 & - & - & 57 & 100 \\
\hline Con alta concentración de capital & 2 & 3.5 & 53 & 93.0 & 2 & 3.5 & 57 & 100 \\
\hline Sin alta concentración de capital & 54 & 94.7 & 1 & 1.8 & 2 & 3.5 & 57 & 100 \\
\hline Redes sencillas & 30 & 52.6 & 23 & 40.4 & 4 & 7.0 & 57 & 100 \\
\hline Redes de redes & 3 & 5.3 & 45 & 78.9 & 8 & 14.0 & 57 & 100 \\
\hline Clusters & - & - & 38 & 68.7 & 17 & 29.8 & 57 & 100 \\
\hline Keiretsus & - & - & 47 & 82.5 & 10 & 17,5 & 57 & 100 \\
\hline Empresa virtual & 3 & 5.3 & 42 & 73.7 & 12 & 21.1 & 57 & 100 \\
\hline
\end{tabular}

* las f (s) indican la frecuencia con que se repite el fenómeno.

Con respecto a la pregunta Estructura legal de los acuerdos (gráfica 3) la más utilizada es el outsourcing (19.3\%) y la franquicia (14\%), resultados que se asemejan a lo apuntado en el ámbito nacional. Sin embargo, 52.6\% aparece bajo la forma de "Otros", es decir, en su carácter legal los acuerdos no se corresponden con las formas tradicionales de los mismos, por lo que posiblemente sea de tipo verbal. 


\section{Gráfica 3}

Estructura legal de los acuerdos

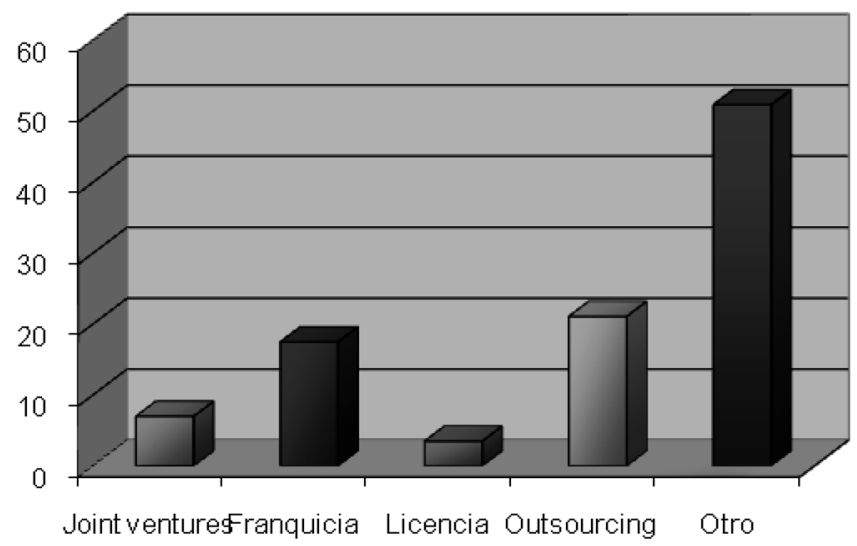

Forma legal de los acuerdos

Fuente: Elaboración propia

Al preguntar sobre Cuáles objetivos se persiguen con las alianzas (cuadro 9), Incrementar el volumen de ventas resultó ser "sumamente importante" para los empresarios merideños (75.4\%), seguido por compartir redes de distribución (42.1\%) como "muy importante". Compartir riesgos (33.3\%) fue considerado como "medianamente importante" y adquirir tecnología (36.8\%) poco importante. Compartir los costes de formación de personal (33.3\%) no fue considerado. Los dos primeros objetivos son comprensibles dada las bajas capacidades de las pequeñas empresas para aumentar los volúmenes vendidos toda vez que al asociarse pueden mejorar las actividades en la cadena de valor, así como contar con canales de distribución para sus productos. 


\section{Cuadro 9}

Objetivos a perseguir con las alianzas

(1=Sumamente importante, $2=$ Muy importante, 3=Medianamente importante, 4=Poco importante, 5=Sin importancia).

\begin{tabular}{|c|c|c|c|c|c|c|c|c|c|c|c|c|c|c|}
\hline \multirow[t]{2}{*}{ Objetivos } & \multicolumn{2}{|c|}{1} & \multicolumn{2}{|c|}{2} & \multicolumn{2}{|c|}{3} & \multicolumn{2}{|c|}{4} & \multicolumn{2}{|c|}{5} & \multicolumn{2}{|c|}{ Ns/nc } & \multicolumn{2}{|c|}{ Total } \\
\hline & $\mathbf{F}$ & $\%$ & f & $\%$ & $\mathbf{F}$ & $\%$ & f & $\%$ & f & $\%$ & f & $\%$ & f & $\%$ \\
\hline Reducir costes & 13 & 22.8 & 3 & 5.3 & 7 & 12.3 & 7 & 12.3 & 9 & 15.8 & 18 & 31.6 & 57 & 100 \\
\hline $\begin{array}{l}\text { Compartir } \\
\text { riesgos }\end{array}$ & 4 & 7.0 & 5 & 8.8 & 19 & 33.3 & 8 & 14.0 & 5 & 8.8 & 16 & 28.1 & 57 & 100 \\
\hline $\begin{array}{l}\text { Adquirir } \\
\text { tecnología }\end{array}$ & 5 & 8.8 & 9 & 15.8 & 5 & 8.8 & 21 & 36.8 & 7 & 12.3 & 10 & 17.5 & 57 & 100 \\
\hline $\begin{array}{l}\text { Mejorar } \\
\text { métodos de } \\
\text { trabajo }\end{array}$ & 10 & 17.5 & 11 & 19.3 & 16 & 28.1 & 9 & 15.8 & 3 & 5.3 & 8 & 14.0 & 57 & 100 \\
\hline $\begin{array}{l}\text { Aumentar } \\
\text { oferta }\end{array}$ & 17 & 29.8 & 14 & 24.6 & 13 & 22.8 & 7 & 12.3 & 4 & 7.0 & 2 & 3.5 & 57 & 100 \\
\hline $\begin{array}{l}\text { Compartir } \\
\text { redes de } \\
\text { distribución }\end{array}$ & 9 & 15.8 & 24 & 42.1 & 4 & 7.0 & 4 & 7.0 & 6 & 10.5 & 10 & 17.5 & 57 & 100 \\
\hline $\begin{array}{l}\text { Incrementar } \\
\text { ventas } \\
\end{array}$ & 43 & 75.4 & 4 & 7.0 & 3 & 5.3 & - & - & - & - & 7 & 12.3 & 57 & 100 \\
\hline $\begin{array}{l}\text { Incrementar \% } \\
\text { de mercado }\end{array}$ & 21 & 36.8 & 12 & 21.1 & 9 & 15.8 & 5 & 8.8 & 3 & 5.3 & 7 & 12.3 & 57 & 100 \\
\hline $\begin{array}{l}\text { Acceder a } \\
\text { mercados }\end{array}$ & 3 & 5.3 & 19 & 33.3 & 13 & 22.8 & 7 & 12.3 & 5 & 8.8 & 10 & 17.5 & 57 & 100 \\
\hline $\begin{array}{l}\text { Compartir } \\
\text { costes de } \\
\text { formar } \\
\text { personal }\end{array}$ & 3 & 5.3 & 2 & 3.5 & 3 & 5.3 & 4 & 7.0 & 19 & 33.3 & 26 & 45.6 & 57 & 100 \\
\hline
\end{tabular}

Nota: Este cuadro se construyó tomando las frecuencias y los porcentajes de los cuadros estadísticos correspondientes a cada uno de los ítems de la pregunta $\mathrm{N}^{\circ} 25$ (de respuesta múltiple) del cuestionario aplicado.

La siguiente pregunta, motivaciones para no asociarse, sólo se formuló a los que se agruparon dentro de la categoría Sin Alianza, con el objeto de dar respuesta a la segunda hipótesis.

Motivaciones para no asociarse. Como se muestra en el cuadro 10, el $69.2 \%$ de los empresarios no desean asociarse porque se considera independiente y $18.3 \%$ no confía en las alianzas. Estas respuestas confirman lo apuntado en el marco teórico en el sentido de que estos aspectos influyen significativamente en el empresario de una Pyme para no realizar asociaciones (Altenburg, 1999; Casani, 1995; Esser et al., 1994; Romero, 2004). 


\section{Cuadro 10}

Motivaciones para no asociarse

\begin{tabular}{l|r|r|r|r}
\hline & Frecuencia & Porcentaje & \multicolumn{1}{c}{$\begin{array}{c}\text { Porcentaje } \\
\text { válido }\end{array}$} & $\begin{array}{c}\text { Porcentaje } \\
\text { acumulado }\end{array}$ \\
\hline $\begin{array}{l}\text { Es independiente, no lo } \\
\text { estima }\end{array}$ & 72 & 69.2 & 69.2 & 69.2 \\
\hline Por falta de información & 2 & 1.9 & 1.9 & 71.2 \\
\hline Teme perder el control & 3 & 2.9 & 2.9 & 74.0 \\
\hline Para evitar la competencia & 4 & 3.8 & 3.8 & 77.9 \\
\hline $\begin{array}{l}\text { Por falta de confianza en las } \\
\text { alianzas }\end{array}$ & 19 & 18.3 & 18.3 & 96.2 \\
\hline Otra & 2 & 1.9 & 1.9 & 98.1 \\
\hline Ns/Nc & 2 & 1.9 & 1.9 & 100.0 \\
\hline Total & 104 & 100.0 & 100.0 & \\
\hline
\end{tabular}

\section{Contrastación de hipótesis}

En esta sección se relacionan variables mediante la aplicación del test de independencia a los cuadros, el cual supone que en la hipótesis nula $\left(\mathrm{H}_{0}\right)$ no hay relación entre las variables. Para contrastar la primera de las hipótesis el estadístico de prueba es una Chi-cuadrado (Lind, Mason y Marchall, 2001) para un nivel de significación del $5 \%$.

$\mathrm{H}_{0}$ : No hay relación entre tamaño de la empresa y alianza

$\mathrm{H}_{\mathrm{I}}$ : Si hay relación entre tamaño de la empresa y alianza

Utilizando una Chi-cuadrado para probar la hipótesis anterior, puesto que $p=0.00<0.05$ se rechaza $\mathrm{H}_{0}$. Por tanto, los resultados de esta investigación indican que el tamaño de la empresa, medido en términos del número de trabajadores de la misma, condiciona las posibilidades de establecer acuerdos (cuadro 11). Sin embargo, la prueba pudiese no considerarse robusta toda vez que una de las frecuencias resultó menor a 5.

\section{Cuadro 11}

\section{Pruebas de chi-cuadrado}

\begin{tabular}{l|c|c|c}
\hline & Valor & Gl & Sig. asintótica (bilateral) \\
\hline Chi-cuadrado de Pearson & 24.216 & 2 & .000 \\
Razón de verosimilitud & 23.662 & 2 & .000 \\
Asociación lineal por lineal & 22.763 & 1 & .000 \\
N de casos válidos & 228 & & \\
\hline
\end{tabular}

Nota: 1 casillas $(16.7 \%)$ tienen una frecuencia esperada inferior a 5. La frecuencia mínima esperada es 2,50. 


\section{Conclusiones}

Es notoria la parcialidad y dispersión de los enfoques que procuran explicar la fundamentación teórica de las alianzas o acuerdos interempresariales; la poca uniformidad con su terminología y conceptualización y la diversidad de criterios para clasificarlos, lo que genera diversas limitaciones de tipo metodológico.

- Baja propensión de las empresas locales a cooperar entre sí.

- Carácter inicial del proceso de cooperación dado el predominio de los acuerdos en el área de marketing.

- Prevalecen los acuerdos de naturaleza vertical, lo cual es entendible por las carencias que presentan las empresas de pequeñas dimensiones en cuanto a insumos o a canales de distribución.

- El objetivo por perseguir con las alianzas resultó ser el incremento en el volumen de ventas, cuya explicación pudiera estar en las limitaciones propias de los pequeños negocios.

- La forma legal más utilizada es la subcontratación (outsourcing) y la franquicia, en correspondencia con lo que ocurre en el nivel nacional.

- Atendiendo a las formas de las alianzas, predominan las no equitativas (none equity strategic alliances) y las coaliciones.

- La falta de confianza y el temor a perder independencia son mencionados como motivaciones para no realizar acuerdos o alianzas.

- Con respecto a la relación entre dimensión de la empresa y conformación de acuerdos, la investigación no es concluyente, por lo cual sería pertinente continuar con la misma aplicando otras metodologías u otras pruebas no paramétricas con el fin de determinar la incidencia de ésta y otras variables en la conformación de acuerdos. Finalmente, profundizar sobre los resultados obtenidos en términos de cuota de mercado alcanzada con la alianza, volumen de ventas, clientes atendidos, entre otras variables.

\section{Referencias}

Altenburg, T. (1999). Pequeñas y medianas empresas en los países en vías de desarrollo. Fomentando su competitividad e integración productiva. Estudios e informes, mayo, Berlín: Instituto de Desarrollo Alemán. 
Berry, A., (1997). SME Competitiveness: The Power of Networking o Subcontracting. Washington, D.C.: IFM.

Camisón, C., (2000). Reflexiones sobre la investigación científica de la PYME, Investigaciones Europeas de Dirección y Economía de la Empresa 2 (2): 13-30.

Casani, F. (1995). La naturaleza de la cooperación empresarial: delimitación del concepto y principales enfoques teóricos, Documentos IADE, Universidad Autónoma de Madrid (36): 67-77.

Coase, R. (1937). The nature of the Firm, Economic Review IV (13): 386-405.

Costa, M., (1989). La cooperación entre empresas, nueva estrategia competitiva. Revista Economía Industrial marzo-abril: 27-45.

Comisión Económica para América Latina (CEPAL) (2007). Cinco piezas de desarrollo productivo. Serie de desarrollo productivo. División de desarrollo productivo. Santiago de Chile: Publicación de N. U. 176.

Corporación de Los Andes (1990). Hacia un nuevo perfil de las PyMes y de las Artes. Mérida, Venezuela.

Eggertsson, T., (1995). El comportamiento económico de las organizaciones. Madrid: Alianza Económica.

Esser, K., W. Hillebrand, D. Messner, y J. Meyer-Stamer (1994). Competitividad sistémica. Competitividad internacional de las empresas y políticas requeridas. Berlín: Instituto Alemán de Desarrollo.

Federación Andaluza de Municipios y Provincias (2004). La cooperación empresarial. Valencia, España.

Freeman, C (1993). El reto de la innovación. La experiencia de Japón. Caracas: Editorial Galac.

Fundación para el desarrollo social (FUNDES) (2002). http://www.fundes.org/ consultado abril 2009. 
García, E. (1993). La cooperación empresarial: una revisión de la literatura. Revista de Economía ICE (714): 87-98.

Hamel, G., I. Doz y C. Prahalad (1989). Colaborate with your competitors- and win. Harvard Business Review 67 (1): 133-139.

Instituto Nacional de Estadística (2009). www.gobiernoenlinea.org.ve, consultado en mayo 2009.

InfoPYME (2000). Organización de las Naciones Unidas para el desarrollo industrial y apoyo a la industria privada, Ianic.utexas.edu/pyme.es, consultado en mayo 2009

Jorde, T. y D. Teece (1989). Competition and cooperation: striking the right balance, California Management Review: 25-37.

Keegan, W. y M. Green (1998). Fundamentos de mercadotecnia internacional. México: Prentice Hall.

Koschatzky, K. (2002). Fundamentos de la economía de redes. Especial enfoque a la innovación. Economía Industrial 4 (346): 15-26.

Lawrence, P. y J. Lorsch (1975). Organización y ambiente. Madrid: Labor.

Lind, D., R. Mason y W. Marchall (2001). Estadística para administración y economía. México: McGraw-Hill.

Mariti, P. y R. Smiley (1983). Co-operative Agreements and the Organization of Industry. The Journal of Industrial Economics 31 (4): 437-451.

Martínez, S. (2001). Aproximación teórica a los acuerdos de cooperación. Documento de trabajo, mayo. Universidad de Cádiz.

Mason, S. (1957). Price Policies and Full Employment, reproducido en E.S. Mason Economy Concentration and the Monopoly Problem, Cambrige, Mass.

Méndez, E. (2003). Una ventana geográfica del Estado Mérida, Alcaldía del Municipio Santos Marquina, Mérida: ULA. 
Menguzzato, M., (1992). La cooperación empresarial. Universidad de Valencia: IMPIVA.

Oficina Internacional del Trabajo, Informe (2004-2005). Las actividades económicas en pequeña escala y la brecha de productividad. www.oit.org/public/ spanish/employment/ consulta noviembre 2007.

Ortiz, I. (2000). Plan estratégico a largo plazo, Mérida estado competitivo 2020. Mérida, Venezuela: Convenio ULA-PDVSA.

Penrose, E. (1959). The Theory of the Growth of the Firm. Oxford: Basil Blackwell.

Piñones, S. (2004). Proyecto regional de cooperación técnica de apoyo a la promoción y desarrollo de alianzas productivas, TCP/RLA/2905 (A). Santiago de Chile: FAO.

Pietrobelli, C. y R. Rabellotti (2005). Mejora de la competitividad en clusters y cadenas productivas en América Latina. El papel de la política. Washington D.C.: Banco Interamericano de Desarrollo: 1-78.

Porter, M. y M. Fuller (1988). Coaliciones y estrategia global. Información comercial española (658): 101-120.

Romero, I. (2004). Las PyMEs en el desarrollo económico. ¿Existe justificación para un sesgo pro PyMEs en las estrategias de desarrollo? VI Reunión de Economía Mundial. Bajadoz, España: 1-26

Rosales, R. (1997). Associativity as a Strategy to Strengthen Small and MediumSized Enterprises. Strategic Industrial Policies Changes (capítulo 51), SELA 7: 1-13

Scheaffer, R., W. Mendenhall y L. Ott (1991). Elementos de Muestreo. México: Grupo Editorial Iberoamérica.

Urra, J. (1998). Una evidencia empírica de los factores subyacentes en la cooperación empresarial. Revista Europea de Dirección y Economía de la Empresa 7 (1): 77-94. 
(1999). Cooperación Interempresarial: Algunas puntualizaciones en una aproximación económica del fenómeno. Revista CEPADE (22): 37-49.

Valdaliso, J. y S. López (2003). Historia económica de la empresa. Madrid: Crítica.

Vázquez, A. (2005). Las nuevas fuerzas del desarrollo. Barcelona: Antoni Bosch Editor. (A) 